\title{
Combination of Cold Atmospheric Plasma and Vitamin C Effectively Disrupts Bacterial Biofilms
}

\author{
Santosh Pandit ${ }^{1}$, Mokkapati VRSS ${ }^{1}$, Saga Huld Helgadóttir ${ }^{2}$, Fredrik Westerlund ${ }^{1}$ and Ivan Mijakovic ${ }^{1,3^{*}}$ \\ ${ }^{1}$ Department of Biology and Biological Engineering, Chalmers University of Technology, Kemivägen 10, SE 412 96, Gothenburg, Sweden \\ ${ }^{2}$ Department of Physics, University of Gothenburg, SE-412 96 Göteborg, Sweden \\ ${ }^{3}$ Novo Nordisk Foundation Center for Biosustainability, Technical University of Denmark, Anger Englundsvej 1, DK 2800 Lyngby, Denmark
}

"Corresponding author: Mijakovic I, Department of Biology and Biological Engineering, Chalmers University of Technology, Kemivägen 10, SE 412 96, Gothenburg, Sweden, Tel: +46-70-982-84-46; E-mail: ivan.mijakovic@chalmers.se

Received date: June 12, 2017; Accepted date: June 27, 2017; Published date: June 30, 2017

Copyright: (c) 2017 Santosh Pandit, et al. This is an open-access article distributed under the terms of the Creative Commons Attribution License, which permits unrestricted use, distribution, and reproduction in any medium, provided the original author and source are credited.

\begin{abstract}
Cold atmospheric plasma (CAP) is increasingly used in medical applications for eradication of bacterial and tumor cells. CAP treatment devices, known as plasma jet pens, produce reactive oxygen and nitrogen species at atmospheric pressure and room temperature. The produced reactive species are concentrated in a small and precisely defined area, allowing for high precision medical treatments. CAP has been demonstrated as very effective against planktonic bacterial cells. Unfortunately, bacterial cells in biofilms are typically aggregated and protected by dense exopolymeric matrix, synthesized and secreted by the bacterial community. The main limitation in using CAP against bacterial biofilms is the thick protective matrix of extracellular polymers that shields bacterial cells within this complex architecture. CAP has also been shown to effectively eradicate tumor cells, but the main current limitation is the susceptibility of the surrounding healthy tissues to higher doses. We have recently demonstrated that vitamin $\mathrm{C}$, a natural food supplement, can be used to destabilize bacterial biofilms and render them more susceptible to the CAP killing treatment. Here we discuss the possible impact that a pre-treatment with vitamin C could have on CAP applications in medicine. Specifically, we argue that vitamin C could enhance the effectiveness of CAP treatments against both the bacterial biofilms and some selected tumors.
\end{abstract}

Keywords: Bacterial biofilms; Vitamin C; CAP

\section{Cold Atmospheric Plasma and Its Medical Applications}

Plasma is defined as the fourth state of matter, but unlike solids, liquids and gasses, it does not occur under normal temperature and pressure conditions. Plasma can be generated from neutral gases such as helium, argon, nitrogen, heliox and atmospheric air by subjecting them to a strong electromagnetic field [1]. In order to be used in medical applications, plasma has to be generated at atmospheric pressure and its temperature must be suitable to treat living tissues (up to $40^{\circ} \mathrm{C}$ at the point of application). This special type of plasma is named cold atmospheric plasma (CAP) [2-4]. CAP is receiving increasing attention in medical applications due to its high bactericidal activity and low toxicity towards healthy human cells [5]. CAP generates reactive oxygen and nitrogen species (RONS), and the oxidative stress caused by RONS is the killing mechanism that damages proteins and nucleic acids [6,7]. The CAP sources typically used for medical treatments are atmospheric pressure plasma jets pens. The Figure 1 shows a schematic representation of CAP composition from a plasma jet pen kINPen ${ }^{\circ} \mathrm{MED}$ (Neoplas Control $\mathrm{GmbH}$, Greifswald, Germany).

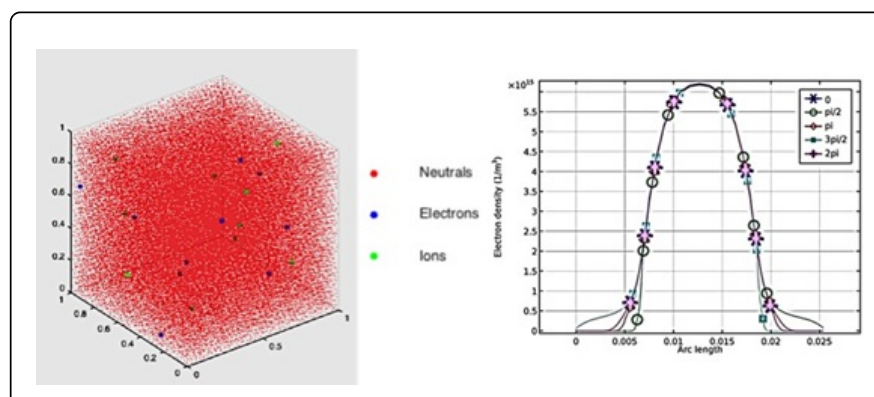

Figure 1: Characteristics of CAP produced by kINPen MED. The left panel shows the distribution of gas with a degree of ionization of $\alpha=10^{-3}$, i.e. containing 10 electrons (blue) and ions (green) for a total of $10^{4}$ particles. The right panel shows the electron density over the plasma gap (arc length) at different times of the production cycle. The electron density is highest in the plasma bulk since the free electrons in the plasma have been accelerated to a sufficiently high kinetic energy to free more electrons from the gas molecules. In addition, the electron density in the sheath region oscillates with the applied electric field.

In dental applications, cold plasma is mostly studied for its antibacterial related activity. In addition to tooth whitening applications, CAP can be used for a painless restoration of dental cavities without drilling, root canal disinfection, elimination of biofilms, bleaching, and treating periodontal and intraoral diseases caused by bacteria [8]. Although heat treatments and antibiotics can also be used to eradicate the bacteria, they cause pain and increase risk 
of antibiotic resistance, respectively, and thus CAP represents a promising alternative. While CAP is used in treatment of root canals and dental caries, it has been noticed that plasma failed to completely eradicate bacterial cells hidden in the deeper layers of the biofilm [9]. Extracellular polymeric substances (EPS) produced by bacterial cell constitute the matrix of the bacterial biofilm, which holds bacterial communities together. The EPS matrix, which is composed of polysaccharides, proteins and extracellular DNA, is known to protect the bacterial communities from various antimicrobial agents, making them several fold more resistant [10,11]. Generally, the dense polymeric matrix is protecting the bacterial cells from antimicrobial exposure by preventing the penetration/diffusion of antimicrobial molecules. Interestingly, the EPS have also been shown to protect bacteria from the CAP treatment [12]. It is evident that long CAP exposure times might have a significant impact on the top layer of cells in bacterial colonies, but hardly any effect on deeper layers.

Outside dentistry, which arguably represents the broadest field of CAP use in medicine, CAP is also used to accelerate the haling of chronic wounds. Chronic wounds, when colonized by bacteria, delay healing and can foster the development of drug-resistant bacteria with prolonged antibiotic treatments. CAP not only enhances the wound healing process but also contains the spread of multi-drug resistant bacteria [13]. Three to five-day course of cold plasma treatment has been shown to be required in order to reduce the bacterial load (Psudomonas aeruginosa) on the wounds [14]. It has been established that, once the plasma treatment course is stopped, wound-healing also slows down [14]. Another in vivo study, using a Micro Plaster alpha device also suggests that wound healing may be accelerated by the CAP treatment [15]. There are studies which provide insights of the mechanism by which the Cap treatment stimulates wound healing. It was reported that CAP treatment influences gene expression of key regulators that are important for inflammation and wound healing, as well as activation of antimicrobial peptides [16]. CAP has been clearly shown to enhance the wound healing effect when used in combination with certain antiseptic liquids [17]. Therefore, CAP is currently in use as a decontaminant in hospital environments [18] and for functionalization of wound dressings [19]. Interestingly, CAP is also being studied in oncology to eradicate tumor cells. It has been demonstrated that cold plasma can induce necrosis, apoptosis and cell detachment by disrupting certain phases of tumor cell replication [20]. CAP effectiveness has been demonstrated against dozens of tumor cell lines and tumor types in vitro and in vivo, respectively [21]. The unfortunate limitation in the use of CAP against tumors is that a high dose CAP exposure can also harm healthy mammalian cells [20], hence the need for precise micro devices. The recent focus in CAPanticancer treatment has thus been on developing small scale and high precision devices (micro capillaries), which led to successful inhibition of tumors [22,23].

\section{Combining Vitamin C with Cold Atmospheric Plasma}

Vitamin C is a common, natural food supplement which does not have any clinical side effects. Vitamin $\mathrm{C}$ has been reported to be extremely toxic to Mycobacterium tuberculosis [24], but not to other common pathogens. There have been reports where vitamin $\mathrm{C}$ can be successfully used to enhance the effect of standard antibiotic treatments $[25,26]$. Our group has examined the effect of vitamin $C$ on bacterial biofilms of that are not killed by vitamin $\mathrm{C}$. We have found that vitamin $\mathrm{C}$ destabilizes their biofilms by inhibiting the production of the matrix EPS (Pandit \& Mijakovic, unpublished results). This leads to reduction in biofilm complexity and it should enhance the susceptibility of cells to various anti-microbial agents. Since CAP treatments are inhibited by the biofilm matrix [12], we have examined whether vitamin C, which inhibits EPS production, could be used to enhance the effectiveness of CAP treatments. We found that the vitamin $\mathrm{C}$ pre-treatment made bacterial biofilms of Bacillus subtilis, Escherichia coli, P. aeruginosa and Staphylococcus epidermidis significantly more prone to extermination by CAP treatments [27]. These findings are summarized in Figure 2. 


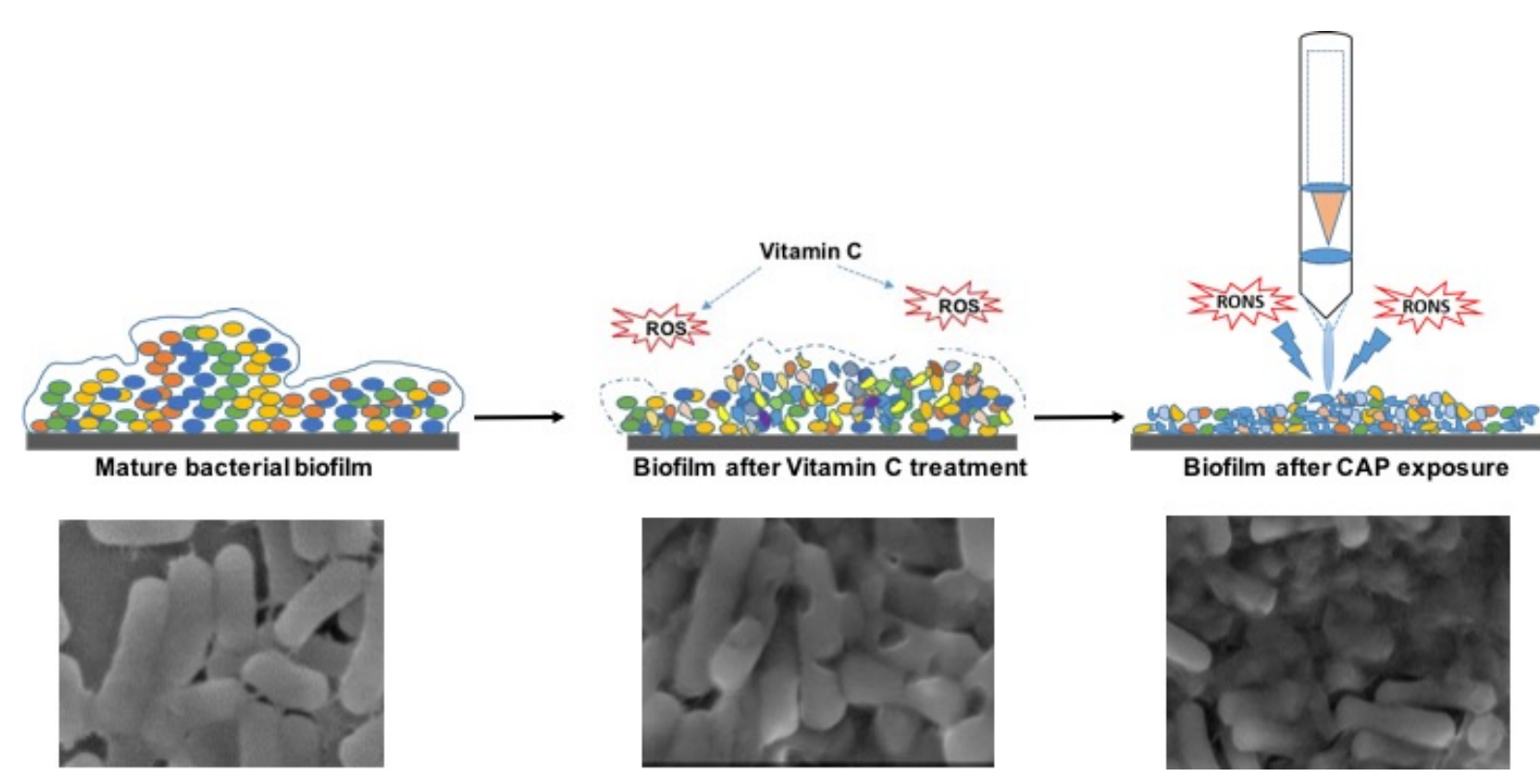

Figure 2: Effect of vitamin C pre-treatment on CAP eradication of a matured bacterial biofilm. The schematics are accompanied with examples of scanning electron micrographs of Bacillus subtilis. The non-treated biofilm is protected by a thick EPS matrix. Vitamin C pre-treatment leads to ROS-induced cellular damage, but mainly it eliminates the production of EPS. The CAP treatment successfully eradicates the exposed bacterial cells by excessive oxidative stress (RONS).

Our results [27] suggest that vitamin $\mathrm{C}$ can be used as an effective pre-treatment, to render CAP treatments more effective. This biofilmdestabilizing effects probably also accounts for previously reported effect of vitamin $\mathrm{C}$ in enhancing common antibiotic treatments, even against bacteria which are not directly killed by vitamin $C[25,26]$. This suggests that vitamin $\mathrm{C}$ could be used as a biofilm destabilizer, to either enhance the efficiently or reduce the exposure time required in antibacterial therapy. Reducing exposure and increasing efficiency is extremely important in capping the growing trend of antibiotic resistance among common bacterial pathogens.

As previously mentioned, CAP is also used for anticancer therapy [20-23]. Vitamin C has also been suggested to have adverse effects on cancer cells [28]. Hypoxia, often encountered in tumors, made multiple cancer cell lines more susceptible to oxidative damage caused by high doses of vitamin C [29]. Some colorectal cancers carry specific mutations that make them specifically sensitive to vitamin C-induced oxidative stress, i.e. they are selective targets for vitamin C [30]. Given the proven anticancer effects of vitamin $\mathrm{C}$, there has been an increased focus on understanding the uptake mechanisms for vitamin $\mathrm{C}$ present in cancer cells [31]. Since CAP and vitamin C both induce ROS and/or RONS formation in tumor cells, it is plausible to presume that a combined treatment could more effectively overrun the defenses against oxidative stress in cancer cells. Therefore we propose that, in addition to enhancing antibacterial effect of CAP, vitamin $C$ could also be an effective enhancer of CAP-based anticancer treatments.

\section{Acknowledgment}

This work was funded by grants from the Chalmers University of Technology and VINNOVA to IM and FW, and Vetenskapsrådet to IM.

\section{References}

1. Hippler R, Kersten H, Schmidt M, Schoenbach KM (2008) Plasma Sources. Low Temperature Plasmas: Fundamentals, Technologies, and Techniques (2nd ed.). Wiley-VCH. ISBN 978-3-527-40673-9.

2. Tendero C, Tixier C, Tristant P, Desmaison J, Leprince P (2006) Atmospheric pressure plasmas: a review. Spectrochim Acta Part B At Spectrosc 61: 2-30.

3. Laroussi M (2009) Low-temperature plasmas for medicine? IEEE Trans. Plasma Sci 37: 714-725.

4. Graves DB (2014) Low temperature plasma biomedicine: a tutorial review. Phys Plasmas 21: 080901.

5. Maisch T, Shimizu T, Li YF, Heinlin J, Karrer S, et al. (2012) Decolonisation of MRSA, S. aureus and E. coli by cold atmospheric plasma using a porcine skin model in vitro. PLoS ONE 7: e34610.

6. Joshi SG, Paff M, Friedman G, Fridman G, Fridman A, et al. (2010) Control of methicillin-resistant Staphylococcus aureus in planktonic form and biofilms: a biocidal efficacy study of nonthermal dielectricbarrier discharge plasma. Am J Infect Control 3: 293-301.

7. de Geyter N, Morent R (2012) Nonthermal plasma sterilization of living and nonliving surfaces. Annu Rev Biomed Eng 14: 255-274.

8. Cha S, Park YS (2014) Plasma in dentistry. Clin Plasma Med 2: 4-10.

9. Arora V, Nikhil V, Suri NK, Arora P (2014) Cold atmospheric plasma (CAP) in dentistry. Dentistry $4: 1$.

10. Branda SS, Chu F, Kearns DB, Losick R, Kolter R (2006) A major protein component of the Bacillus subtilis biofilm matrix. Mol Microbiol 59: 1229-38.

11. Barnes AM, Ballering KS, Leibman RS, Wells CL, Dunny GM (2012) Enterococcus faecalis produces abundant extracellular structures containing DNA in the absence of cell lysis during early biofilm formation. MBio 3: e00193-12.

12. Mai-Prochnow A, Bradbury M, Ostrikov K, Murphy AB (2015) Pseudomonas aeruginosa biofilm response and resistance to cold atmospheric pressure plasma is linked to the redox-active molecule phenazine. PLoS ONE 10: e0130373. 
Citation: Pandit S, Mokkapati VRSS, Helgadóttir SH, Westerlundan F, Mijakovic I (2017) Combination of Cold Atmospheric Plasma and Vitamin C Effectively Disrupts Bacterial Biofilms. Clin Microbiol 6: 283. doi:10.4172/2327-5073.1000283

Page 4 of 4

13. Daeschelin G, Napp M, Lutze S, Arnold A, von podewils S, et al. (2015) Skin and wound decontamination of multidrug-resistant bacteria by cold atmospheric plasma coagulation. J Dtsch Dermatol Ges 13: 143-150.

14. Ermolaeva SA, Varfolomeev AF, Chernukha MY, Yurov DS, Vasiliev MM, et al. (2011) Bactericidal effects of non-thermal argon plasma in vitro, in biofilms and in the animal model of infected wounds. J Med Microbiol 60: 75-83.

15. Isbary G, Stolz W, Shimizu T, Monetti R, Bunk W, et al. (2013) Cold atmospheric argon plasma treatment may accelerate wound healing in chronic wounds: Results if an open retrospective randomized controlled study in vivo. Clin Plasma Med 1: 25-30.

16. Arndt S, Landthaler M, Zimmerman JL, Unger P, Wacker P, et al. (2015) Effects of cold atmospheric plasma (CAP) on ß-defensins, inflammatory cytokines, and apoptosis-related molecules in keratinocytes in vitro and in vivo. PLoS one 3: 1-16.

17. Klebes M, Ulrich C, Kluschke F, Patzelt A, Vandersee S, et al. (2014) Combined antibacterial effects of tissue-tolerable plasma and a modern conventional liquid antiseptic on chronic wound treatment. J Biophotonics. 8: 382-391.

18. Cahill OJ, Claro T, O’Connor N, Cafolla AA, Stevens NT, et al. (2014) Cold air plasma to decontaminate inanimate surfaces of the hospital environment. Appl Environ Microbiol 80: 2004-2010.

19. Spange S, Pfuch A, Wirgand C, Beier O, Hipler UC, et al. (2015) Atmospheric pressure plasma CVD as a tool to functionalise wound dressings. J Mater Sci: Mater Med 26: 76.

20. Hoffmann C, Berganza C, Zhang J (2013) Cold atmospheric plasma: methods of production and application in dentistry and oncology. Med Gas Res 3: 21.

21. Yan D, Sherman JH, Keidar M (2017) Cold atmospheric plasma, a nove promising anti-cancer treatment modality. Oncotarget 8: 15977-15995.
22. Chen Z, Simonyan H, Cheng X, Gjika E, Lin L, et al. (2017) A novel micro cold atmospheric plasma device for glioblastoma both in vitro and in vivo. Cancers (Basel) 9: E61.

23. Binenbaum Y, Ben-David G, Gil Z, Slutsker YZ, Ryzhkov MA, et al. (2017) Cold atmospheric plasma, created at the tip of an elongated flexible capillary using low electric current, can slow the progression of melanoma. PLoS One 12: e0169457.

24. Vilchèze C, Hartman T, Weinrick B, Jacobs WR Jr (2013) Mycobacterium tuberculosis is extraordinarily sensitive to killing by a vitamin $\mathrm{C}$-induced Fenton reaction. Nat Commun 4: 1881.

25. Khameneh B, Fazly Bazzaz BS, Amani A, Rostami J, Vahdati-Mashhadian $\mathrm{N}$ (2016) Combination of anti-tuberculosis drugs with vitamin C or NAC against different Staphylococcus aureus and Mycobacterium tuberculosis strains. Microb Pathog 93: 83-87.

26. Sezikli M, Çetinkaya ZA, Güzelbulut F, Yeşil A, Coşgun S, et al. (2012) Supplementing vitamins $\mathrm{C}$ and $\mathrm{E}$ to standard triple therapy for the eradication of Helicobacter pylori. J Clin Pharm Ther 37: 282-285.

27. Helgadóttir S, Pandit S, Mokkapati VRSS, Westerlund F, Apell P, et al. (2017) Vitamin C pretreatment enhances the antibacterial effect of cold atmospheric plasma. Front Cell Infect Microbiol 7: 43.

28. Park C (2013) The effects of high concentrations of vitamin C on cancer cells. Nutrients 5: 3496-3505.

29. Tian W, Wang Y, Xu Y, Guo X, Wang B, et al. (2014) The hypoxiainducible factor renders cancer cells more sensitive to vitamin $\mathrm{C}$-induced toxicity. J Biol Chem 289: 3339-3351.

30. Yun J, Mullarky E, Lu C, Bosch KN, Kavalier A, et al. (2015) Vitamin C selectively kills KRAS and BRAF mutant colorectal cancer cells by targeting GAPDH. Science 350: 1391-1316.

31. Wohlrab C, Phillips E, Dachs GU (2017) Vitamin C transporters in cancer: current understanding and gaps in knowledge. Front Oncol 7: 74. 